\title{
INFRARED SPECTROSCOPY OF SYMBIOTIC STARS. X. ORBITS FOR THREE S-TYPE SYSTEMS: V1044 CENTAURI, HEN 3-1213, AND SS 73-96
}

\author{
Francis C. Fekel ${ }^{1,4}$, Kenneth H. Hinkle ${ }^{2}$, Richard R. Joyce ${ }^{2}$, and Peter R. Wood ${ }^{3}$ \\ ${ }^{1}$ Tennessee State University, Center of Excellence in Information Systems, 3500 John A. Merritt Boulevard, \\ Box 9501, Nashville, TN 37209 USA; fekel@evans.tsuniv.edu \\ ${ }^{2}$ National Optical Astronomy Observatory, P.O. Box 26732, Tucson, AZ 85726 USA; hinkle@noao.edu, joyce@noao.edu \\ ${ }^{3}$ Research School of Astronomy and Astrophysics, Mount Stromlo Observatory, Australian National University, \\ Cotter Road, Weston Creek, ACT 2611, Australia; wood@mso.anu.edu.au \\ Received 2015 April 2; accepted 2015 June 10; published 2015 July 15
}

\begin{abstract}
Employing new infrared radial velocities, we have computed orbits of the cool giants in three southern S-type symbiotic systems. The orbit for V1044 Cen, an M5.5 giant, has a period of 985 days and a modest eccentricity of 0.16 . Hen 3-1213 is a K4 giant, yellow symbiotic with an orbital period of 533 days and a similar eccentricity of 0.18 . For the M2 giant SS 73-96 the orbital period is 828 days, and this system has a somewhat larger eccentricity of 0.26 . Measurement of the $\mathrm{H}_{\mathrm{I}}$ Paschen $\delta$ emission lines, which may at least partially reflect the motion of the secondary in SS 73-96, results in a mass ratio of 2.4 for the $M$ giant relative to the presumed white dwarf. The estimated orbital inclinations of V1044 Cen and Hen 3-1213 are low, about 40 . However, for SS 73-96 the predicted inclination is $90^{\circ}$, and so an ephemeris for eclipses of the secondary or the hot nebula surrounding it is provided. A search of the orbital velocity residuals of V1044 Cen and SS 73-96 for pulsation periods produced no realistic or convincing period for either star.
\end{abstract}

Key words: binaries: symbiotic - infrared: stars - stars: individual (V1044 Cen, Hen 3-1213, SS 73-96) - stars: late-type

\section{INTRODUCTION}

Symbiotic binaries consist of evolved cool giants, usually of spectral class $\mathrm{M}$, that transfer mass to their companions. In the visible the spectrum is a combination of the stellar spectrum plus emission lines from an ionized nebula. The binary secondary is typically a compact object, usually a white dwarf although at least in one case (Hinkle et al. 2006) it is a neutron star. In principle the secondary could be a low mass, mainsequence star. Observations at infrared wavelengths by Webster \& Allen (1975) led to the separation of the symbiotics into two subclasses, D for dusty-type, which has a dust shell that often obscures the stellar spectrum, and S for stellar-type systems that have no evidence of dust. The S-type symbiotics mostly have orbital periods in the range of $1-5$ years. The D-type systems contain Mira variables (e.g., Whitelock 1987), and because of the size of such objects, those systems have periods that are at least an order of magnitude longer than their S-type cousins (Schmid \& Schild 2002; Hinkle et al. 2013). Instead of an $\mathrm{M}$ giant, a small number of symbiotics contain $\mathrm{F}$, $\mathrm{G}$, and $\mathrm{K}$ giants, and these systems are called yellow symbiotics (Allen et al. 1982; Schmid \& Nussbaumer 1993). For a more extensive introduction to symbiotic stars see Fekel et al. (2000) and Mikołajewska (2003).

An understanding of symbiotic binaries begins with a determination of the orbital period and other orbital elements of the late-type giant. Gromadzki et al. (2013) analyzed symbiotics listed in Belczyński et al. (2000) that were observed in the large photometric surveys of the All Sky Automated Survey (ASAS), MACHO, and OGLE and estimated their orbital periods. As a result of this and previous work, orbital

\footnotetext{
${ }^{4}$ Visiting Astronomer, Kitt Peak National Observatory, National Optical Astronomy Observatory, operated by the Association of Universities for Research in Astronomy, Inc., under cooperative agreement with the National Science Foundations.
}

periods now have been estimated for more than $50 \%$ of the S-type systems (Mikołajewska 2012) in the catalog of Belczyński et al. (2000). Spectroscopic observations to detect the cool giant component are best made at infrared wavelengths because at blue wavelengths nebular emission contaminates the spectrum. Another difficulty is the faintness of the stars. About $50 \%$ of the confirmed symbiotics in the catalog of Belczyński et al. (2000) have $V \geqslant 13.0$ mag. Thus, moderate aperture telescopes are required to acquire high-resolution spectra from which precise velocities can be measured. Unfortunately, the long orbital periods of the systems result in low velocity amplitudes. In addition, pulsational velocity changes contaminate the orbital velocity variations, increasing the velocity residuals to orbital fits. These problems make it difficult to obtain well-determined orbital elements. Currently, spectroscopic orbits for about 40 of the cool giant components in the S-type symbiotics have been determined (Mikołajewska 2011; Jorissen et al. 2012). Thus, of the nearly 200 symbiotic systems listed in the catalog of Belczyński et al. (2000), at present only about $20 \%$ have had spectroscopic orbital elements determined for the cool giant component. While less than 300 symbiotic systems are currently known in the Milky Way (Miszalski et al. 2013), spectroscopic searches to identify new symbiotics, especially in the Galactic Bulge, have had good success (Miszalski et al. 2013; Miszalski \& Mikołajewska 2014).

In eight previous papers in this series, the most recent being Fekel et al. (2010), we have determined the orbital elements for the late-type component in 24 systems, nine of which are first orbits.

The present work provides the spectroscopic orbits for the late-type giant components in three S-type symbiotics, V1044 Cen, Hen 3-1213, and SS 73-96. Unless a system has a variable star designation, the name with which it is identified usually comes from one of several survey lists because of the faintness of the objects in this class. The catalog of Belczyński et al. 
Table 1

Basic Properties of the Program Stars

\begin{tabular}{|c|c|c|c|c|c|c|}
\hline Name & $\begin{array}{c}V \\
(\mathrm{mag})\end{array}$ & $\begin{array}{c}K \\
(\mathrm{mag})\end{array}$ & $\begin{array}{l}H-K \\
(\mathrm{mag})\end{array}$ & $\begin{array}{c}\text { Primary } \\
\text { Spectral Class }\end{array}$ & $\begin{array}{c}\text { Orbital Period } \\
\text { (days) }\end{array}$ & $\begin{array}{c}\dot{\mathrm{M}} \\
\left(M_{\odot} \mathrm{yr}^{-1}\right)\end{array}$ \\
\hline V1044 Cen & $11.15^{\mathrm{b}}$ & $5.43^{b}$ & $0.26^{\mathrm{b}}$ & M5. $5^{\mathrm{c}}$ & 985 & $\ldots$ \\
\hline Hen 3-1213 & $12.78^{\mathrm{d}}$ & $6.99^{\mathrm{e}}$ & $0.31^{\mathrm{e}}$ & $\mathrm{K} 4^{\mathrm{f}}$ & 533 & $\ldots$ \\
\hline SS 73-96 & $15.24^{\mathrm{b}}$ & $6.36^{\mathrm{b}}$ & $0.44^{\mathrm{b}}$ & $M 2^{f}$ & 828 & $2.1 \times 10^{-7 a}$ \\
\hline
\end{tabular}

References: ${ }^{\mathrm{a}}$ Seaquist et al. (1993), ${ }^{\mathrm{b}}$ Munari et al. (1992), ${ }^{\mathrm{c}}$ Mürset \& Schmid (1999), ${ }^{\mathrm{d}}$ Gutierrez-Moreno et al. (1999), ${ }^{\mathrm{e}}$ Phillips (2007), ${ }^{\mathrm{f}}$ Allen (1980).

(2000) provides a variety of alternative designations for known symbiotics.

Being located in the southern sky, these symbiotic stars have not been as extensively observed and analyzed as most of their northern brethren. It is hoped that the determination of the orbits and the discussion in this paper will lead to more intensive observing of these symbiotics. Some basic information for the three systems is provided in Table 1.

\section{OBSERVATIONS AND REDUCTIONS}

From 2001 March through 2002 December we acquired spectrograms of the three southern stars with the $1.88 \mathrm{~m}$ telescope and coudé spectrograph system at the Mount Stromlo Observatory (MSO), Canberra, Australia. The detector was an infrared camera, NICMASS, developed at the University of Massachusetts. We obtained a two pixel resolving power of 44000 at a wavelength of $1.623 \mu \mathrm{m}$. Joyce et al. (1998) and Fekel et al. (2000) have provided a more complete description of the experimental setup. The detector and electronics were previously used at Kitt Peak National Observatory (KPNO) with the coudé feed telescope and spectrograph for our northern symbiotics survey.

Unfortunately, both the $1.88 \mathrm{~m}$ telescope at MSO and our infrared NICMASS camera were destroyed by the major Canberra area bush fires of 2003 January. This led to the premature conclusion of our observing program at that observatory.

Between 2003 February and 2010 June we obtained a small number of additional spectra with the Phoenix cryogenic echelle spectrograph, mounted on the $8 \mathrm{~m}$ Gemini South telescope at Cerro Pachon, Chile. A complete description of the spectrograph can be found in Hinkle et al. (1998). The Gemini South observations were centered at various infrared wavelengths including 1.563, 2.226, and $2.364 \mu \mathrm{m}$. Those infrared spectra have a resolving power of either 50000 or 70000 .

Despite their southern declinations of about $-37^{\circ}$, after the loss of the telescope and infrared camera at MSO we were able to acquire a number of additional spectrograms of V1044 Cen and SS 73-96 at KPNO, which at latitude $+32^{\circ}$ is situated well above the equator in the northern hemisphere. However, at a declination of nearly $-52^{\circ}$ Hen 3-1213 rises only $6^{\circ}$ above the horizon at KPNO, and no attempt was made to observe it from there. The KPNO observations, spanning 2004 May to 2014 June, were carried out using the $0.9 \mathrm{~m}$ coudé feed telescope, coudé spectrograph, and a CCD, designated LB1A. This $1980 \times 800$ pixel CCD was manufactured by Lawrence Berkeley National Laboratory and is $300 \mu \mathrm{m}$ thick. Our spectrograms, centered near $1.005 \mu \mathrm{m}$, have a wavelength range of $420 \AA$, and a resolving power of $\sim 21500$.

A few additional observations for our project were made with the $1.5 \mathrm{~m}$ telescope at the Cerro Tololo Inter-American
Observatory. That telescope is operated by the Small and Moderate Aperature Research Telescope System (SMARTS) consortium of universities and other organizations. From 2009 May through 2010 May we obtained a total of six spectra with that telescope, a fiber fed echelle spectrograph, and a $2 \mathrm{~K} \mathrm{SITe}$ CCD. The spectra have a resolving power of $\sim 25000$ at $5500 \AA$.

We have used standard observing and reduction techniques (Joyce 1992). Unfortunately, the spectral coverage at infrared wavelengths 1.563 and $2.226 \mu \mathrm{m}$ was far too small to include a sufficient number of ThAr emission lines for a wavelength calibration solution. Instead, at $1.563 \mu \mathrm{m}$ we utilized the absorption lines of a bright late-type giant star, usually $\delta$ Oph. Approximately 20 lines of $\mathrm{CN}, \mathrm{Fe}$, and $\mathrm{OH}$ were used to calculate the dispersion solution in this wavelength region. Each solution was offset to an absolute standard provided by one or more telluric $\mathrm{OH}$ emission lines. At $2.226 \mu \mathrm{m}$ the wavelength calibration was done by measuring telluric absorption lines in the spectrum of $\beta$ Lup, a bright early-type star. The typical rms of the solutions for both regions was about $0.02 \AA$.

For the spectrograms acquired with the LB1A CCD at $1.005 \mu \mathrm{m}$, and the optical wavelength echelle spectrograms from the $1.5 \mathrm{~m}$ SMARTS telescope, we were able to use ThAr spectra for wavelength calibration. Significant telluric lines occur in the 1.005 and $2.226 \mu \mathrm{m}$ wavelength regions. These lines were removed from our observations by ratioing the spectra to a hot star spectrum observed on the same night.

The wavelength range covered by the echelle spectrograms from the $1.5 \mathrm{~m}$ SMARTS telescope is 4020-7300 $\mathrm{A}$. In symbiotic binaries an emission continuum and emission lines from the gas around the secondary make it difficult if not impossible to measure the late-type giant absorption line spectrum at blue and visual wavelengths. Thus, we measured velocities around $7130 \AA$, which is one of the two reddest orders of the echelle spectrum. Because telluric lines are generally weak in this region, we did not remove them from the measured spectra.

Representative spectra of the program stars at wavelengths of $1.005,1.563$, and $2.226 \mu \mathrm{m}$ are shown in Figures 1-3, respectively. No spectra of Hen 3-1213 were obtained at $1.005 \mu \mathrm{m}$, and so Figure 1 shows the spectra of only V1044 Cen and SS 73-96. Spectra of $\delta$ Oph, our primary velocity standard, which has a spectral type of M0.5 III (Keenan \& McNeil 1989), have been included for comparison in the three figures. In all three regions the vast majority of the lines arise in the photosphere of the red giant. See Fekel et al. (2010) for a summary of the molecular and atomic lines in the spectra of the various regions.

In the redder spectral regions that we have observed there are no emission lines. However, the spectrum of SS 73-96 in the 


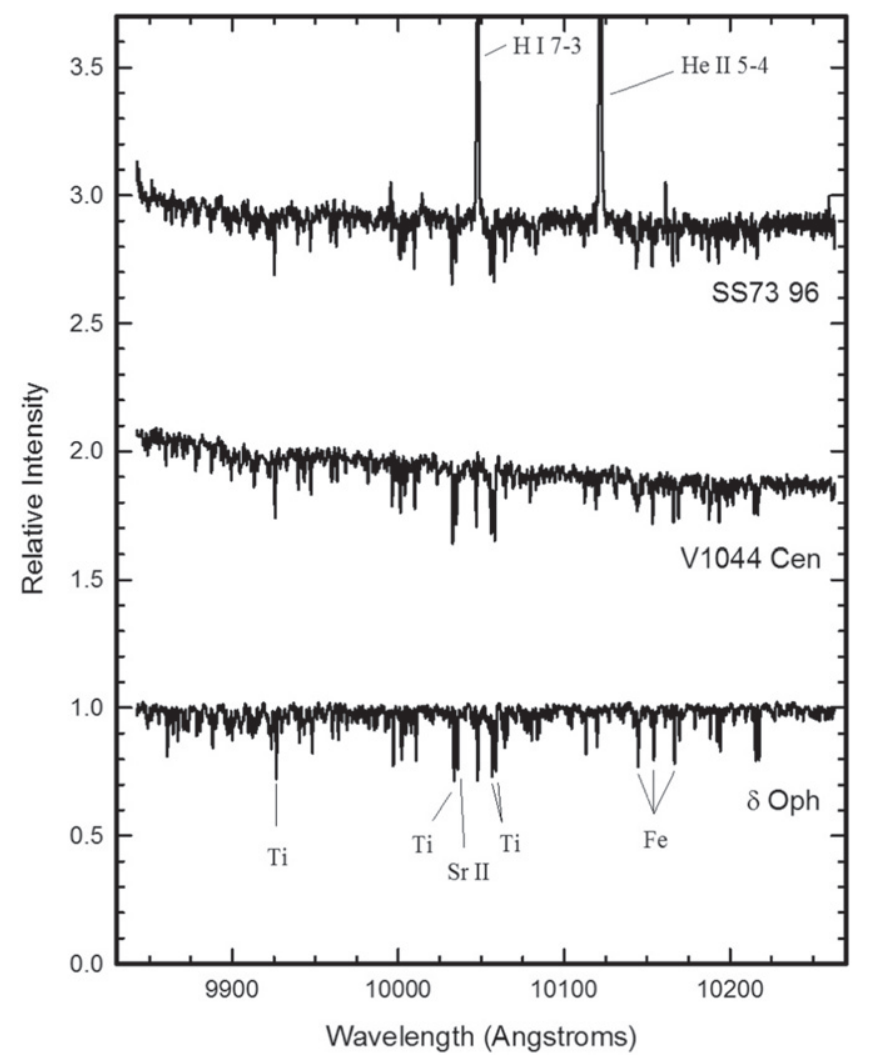

Figure 1. Spectra of V1044 Cen and SS 73-96 and velocity standard $\delta$ Oph at $1.005 \mu \mathrm{m}$ observed with the KPNO coudé feed telescope, spectrograph, and LB1A CCD. The entire observed spectral region is shown. The telluric spectrum has been ratioed out by referencing a hot star spectrum acquired on the same night. The relative intensity scales for V1044 Cen and SS 73-96 have been offset by 1.0 , and 2.0, respectively. A few of the stronger representative absorption and emission lines are identified.

$1.005 \mu \mathrm{m}$ region shows two relatively strong emission features, the $\mathrm{H}_{\mathrm{I}}$ Paschen $\delta$ line at $1.004938 \mu \mathrm{m}$ and the He II line at $1.012361 \mu \mathrm{m}$.

The IRAF cross-correlation program FXCOR (Fitzpatrick 1993) was used to measure the absorption line radial velocities of the program stars. The $\mathrm{M}$ giant IAU radial velocity standard $\delta$ Oph, which was observed multiple times during the course of most nights was normally used as the reference star. Its radial velocity of $-19.1 \mathrm{~km} \mathrm{~s}^{-1}$ was adopted from the work of Scarfe et al. (1990). If $\delta$ Oph was not observed, several other $M$ giants were used as velocity standards. From our unpublished observations of those stars we adopted velocities of $21.3 \mathrm{~km} \mathrm{~s}^{-1}$ for $\mathrm{HR} 3718,18.0 \mathrm{~km} \mathrm{~s}^{-1}$ for $\mathrm{HR} 4162$, $58.7 \mathrm{~km} \mathrm{~s}^{-1}$ for $\mathrm{HR} 5181,-9.6 \mathrm{~km} \mathrm{~s}^{-1}$ for $\mathrm{HR} 7900$, and $-22.2 \mathrm{~km} \mathrm{~s}^{-1}$ for HR 7951 .

The Paschen $\delta$ and He II emission line radial velocities were measured with the IRAF task RVIDLINES. A Gaussian function was fitted to each emission line, and the wavelength shift of the spectral feature relative to its rest wavelength was converted into a radial velocity, which was then corrected for the Earth's motion.

\section{ORBITAL ANALYSIS}

We searched for the orbital periods of the giants with a program named PGRAM (PeriodoGRAM), which compares the phased velocities for each trial period with a sine curve fit. The sum of the squared velocity residuals from the fit was

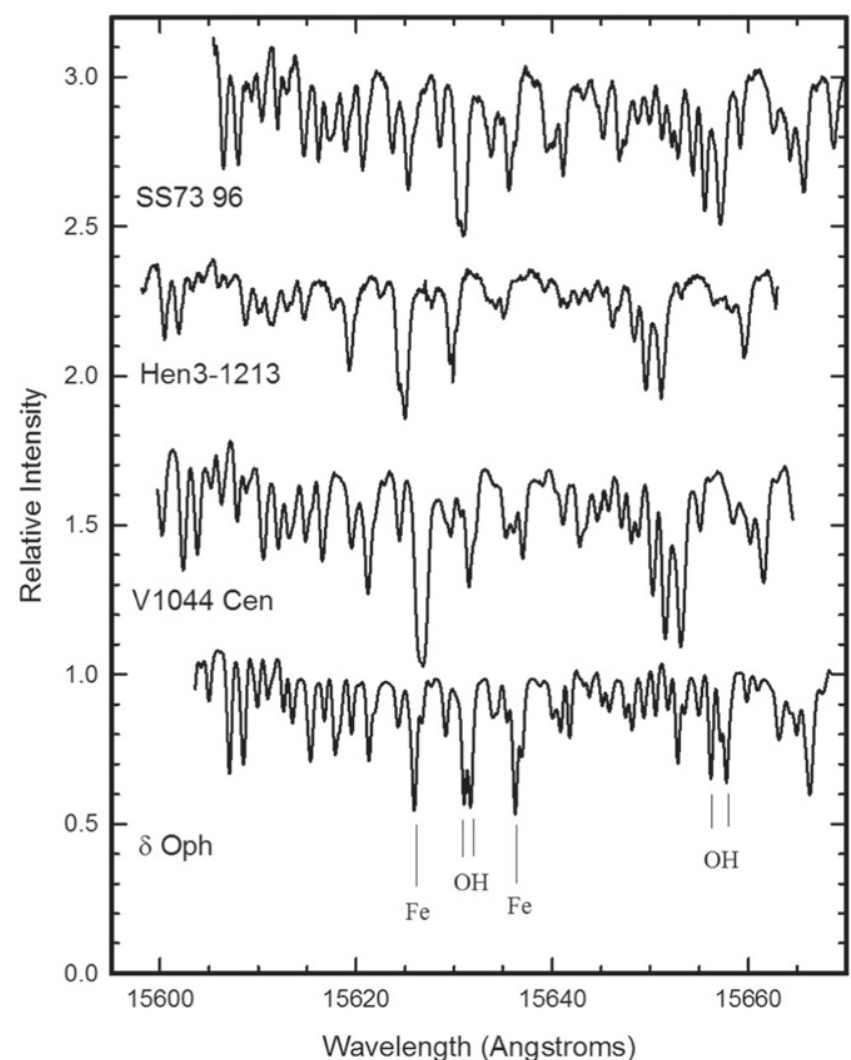

Figure 2. Spectra of V1044 Cen, Hen 3-1213, SS 73-96, and $\delta$ Oph at $1.563 \mu \mathrm{m}$ observed with the Phoenix spectrograph on the Gemini South telescope. The relative intensity scales for V1044 Cen, Hen 3-1213 and SS 7396 have been offset by $0.66,1.33$, and 2.0 , respectively. No correction for telluric absorption lines is needed in this spectral region.

computed, and the period having the smallest value of that sum was chosen as the best preliminary period. Adopting this initial period, spectroscopic orbital elements of each system were determined with various computer programs (Fekel et al. 2008) for eccentric and circular orbits.

\section{PULSATION PERIOD SEARCH}

All $\mathrm{M}$ giants have light variability from pulsation, and the amplitude of that variability usually increases with later spectral type and higher luminosity (Percy et al. 2001). Variables with amplitudes less than $2.5 \mathrm{mag}$ are called semiregular. Lebzelter \& Hinkle (2002) noted that the periods of these semi-regular variables typically range from 30 to 200 days. Velocity variations in semi-regular variables accompany the light variability and generally display amplitudes of up to a few $\mathrm{km} \mathrm{s}^{-1}$ (e.g., Lebzelter \& Hinkle 2002). Thus, for our two symbiotic binaries with $\mathrm{M}$ spectral classes, a portion of the radial velocity variations likely results from pulsation.

After determining orbital elements for those two red giants, we also performed period searches of the residuals of our adopted absorption-line radial velocity solutions with PGRAM. However, we caution that the number and distribution of our observations is not well suited to identifying pulsation periods. This is because our velocity observations, two or sometimes three per year for 9-13 years, are not particularly well spaced to determine periods of weeks or several months. In addition, the low amplitudes, only about $2 \mathrm{~km} \mathrm{~s}^{-1}$, of our best possible pulsation periods are often dependent on the placement of the 


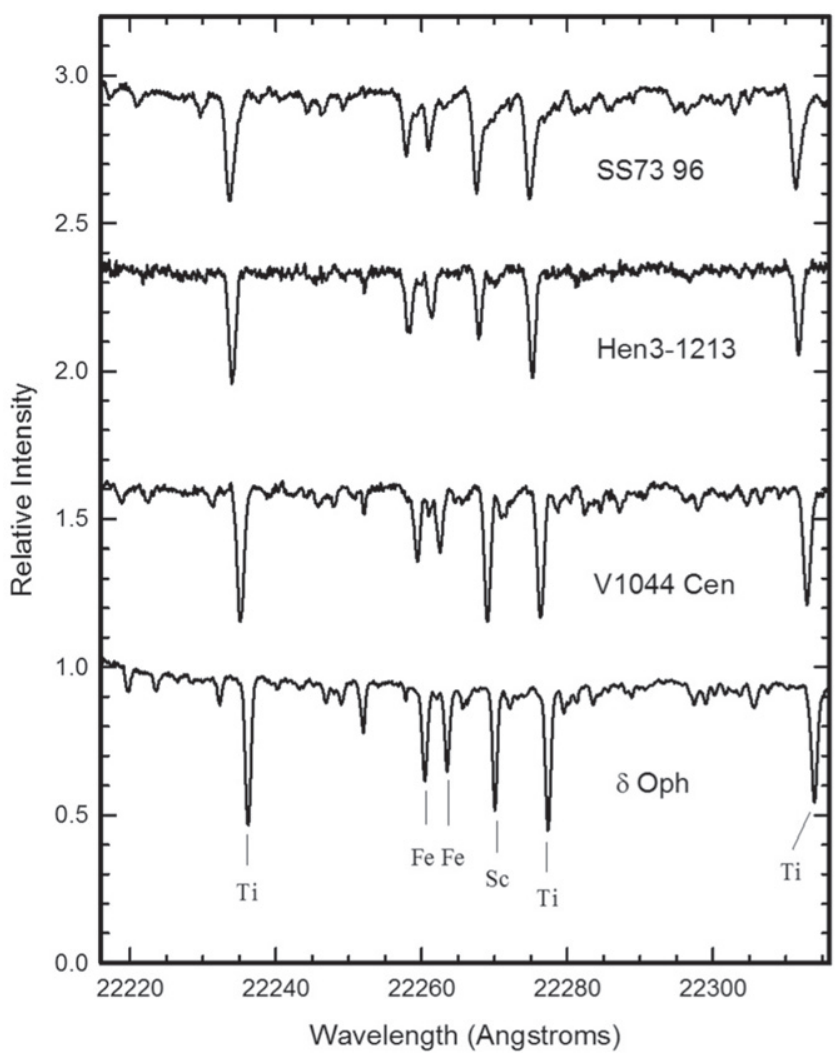

Figure 3. Spectra of V1044 Cen, Hen 3-1213, SS 73-96 and $\delta$ Oph at $2.226 \mu \mathrm{m}$ observed with the Phoenix spectrograph on the Gemini South telescope. The relative intensity scales for V1044 Cen, Hen 3-1213, and SS 73-96 have been offset by $0.66,1.33$, and 2.0 , respectively. The telluric spectrum has been ratioed out by referencing a hot star spectrum acquired on the same night.

few velocities with the largest residuals. Nevertheless, in the sections for the individual stars we discuss our specific results.

\section{ROTATIONAL VELOCITY}

Fekel et al. (2003) measured the FWHM of several isolated atomic features in the $2.226 \mu \mathrm{m}$ region. They then used a empirical broadening relation to convert those results into $v \sin i$ values. We have used the same procedure to determine the projected rotational velocities of our three stars.

\section{V1044 CEN $=$ CD $-368436=$ HEN 3-886 = NSV 6160 \\ 6.1. Short History}

Examining their objective prism spectra, Bidelman \& MacConnell (1973) identified a variety of bright stars of astrophysical interest in the southern sky. They included CD -36 8436 in a list of M-type Mira variables. From data taken by the ASAS program (Pojmanski 2002), Wils (2003) estimated a $V$ magnitude range of $10.4-10.9$, a light variability period of 101: days, and an SR: variability type, with the colon indicating that CD -36 8436 is either a semi-regular or an irregular variable. As a result, Kazarovets et al. (2003) assigned CD -36 8436 the variable star name V1044 Cen, which we use to identify the system in the rest of this paper.

From an objective prism survey to find southern emission line stars and a follow-up spectrum acquired by Carlson (1968), Henize (1976) called V1044 Cen a probable symbiotic star. In his catalog of symbiotic stars Allen (1984) remained cautious and only listed it as a possible member. GutierrezMoreno et al. (1992) obtained spectra of V1044 Cen that showed TiO absorption bands and high-excitation emission lines, confirming that it is a symbiotic star. Munari \& Patat (1993) included the star in a search for resolved $\mathrm{H} \alpha$ nebulae around symbiotic stars, but found its stellar image to be pointlike with no extended nebulosity. Munari et al. (1992) acquired optical and infrared photometry of 93 symbiotic binaries including V1044 Cen. Mürset \& Schmid (1999) estimated a spectral class of M5.5 from an analysis of the strength of several near-infrared wavelength $\mathrm{TiO}$ bandheads. Zamanov et al. (2007) determined an average $v$ sin $i$ value of $7.6 \mathrm{~km} \mathrm{~s}^{-1}$ from two different methods of measurement.

\subsection{Spectroscopic Orbit}

Between 2001 March and 2014 June we acquired 45 observations of V1044 Cen at four observatories (Table 2). Our initial search of the velocities produced a period of 985 days. Determination of the full set of orbital elements resulted in a period of $985.0 \pm 5.4$ days and an eccentricity of $0.16 \pm 0.06$. The uncertainty in the eccentricity is large enough so that we also computed a circular orbit for comparison. The tests of Lucy \& Sweeney (1971) indicate that the eccentric orbit is to be preferred, and so the orbital elements for this solution are given in Table 3. Figure 4 compares our measured radial velocities and the computed radial velocity curve. Zero phase is a time of periastron.

\subsection{Emission Line Spectrum}

Symbiotics in general display many strong emission lines (e.g., Allen 1984; Gutierrez-Moreno et al. 1999) from an ionized nebula (Mikołajewska 2003), and several nebular regions are usually postulated including one surrounding the hot component (e.g., Dobrzycka et al. 1993). Thus, it is possible that some emission lines may at least partially reflect the motion of the secondary.

Gutierrez-Moreno et al. (1992) obtained a spectrum of V1044 Cen that covers a wavelength range of approximately $3600-7100 \AA$ with a resolution of about $5 \AA$. Their spectrum showed the Balmer lines to be in emission. However, emission lines from other elements were rather weak or missing. For example, the $\mathrm{He}_{\mathrm{I}}$ line at $5876 \AA$ was not very strong and $\mathrm{He}$ II at $4686 \AA$ was barely visible.

We examined our spectra of the $1 \mu \mathrm{m}$ region for the Paschen $\delta$ and He II emission lines, which are seen in a number of the symbiotic binaries that we have observed such as V455 Sco, SS 73-90, AE Ara, and SS 73-147 (Fekel et al. 2008, 2010). However, we found the Paschen $\delta$ line of V1044 Cen to be a significant absorption feature in our spectra. Radial velocity measurements of that line in spectra having phases close to maximum and minimum orbital velocity indicate that the absorption line comes from the $\mathbf{M}$ giant component. In addition, no He II emission lines or any other emission features are detected in this region of the spectrum (see Figure 1).

\subsection{Pulsation Period Search}

The light curves of symbiotic stars often show several different variations such as irregularly occuring nova-like outbursts from the hot component as well as radial pulsations or semi-regular variations from the cool star. Evidence of binary motion is produced by eclipses, the reflection effect, and 
Table 2

Absorption Line Radial Velocities of V1044 Cen

\begin{tabular}{|c|c|c|c|c|}
\hline $\begin{array}{l}\text { HJD } \\
-2400000\end{array}$ & Phase & $\begin{array}{l}\text { Velocity } \\
\left(\mathrm{km} \mathrm{s}^{-1}\right)\end{array}$ & $\underset{\left(\mathrm{km} \mathrm{s}^{-1}\right)}{O-C}$ & Observatory \\
\hline 51990.155 & 0.737 & -52.1 & 0.9 & MSO \\
\hline 52044.056 & 0.792 & -53.6 & 0.6 & MSO \\
\hline 52092.966 & 0.841 & -56.2 & -1.0 & MSO \\
\hline 52131.988 & 0.881 & -55.2 & 0.5 & MSO \\
\hline 52315.142 & 0.067 & -50.6 & 1.8 & MSO \\
\hline 52349.166 & 0.101 & -51.6 & -0.5 & MSO \\
\hline 52353.049 & 0.105 & -52.1 & -1.1 & MSO \\
\hline 52398.111 & 0.151 & -51.8 & -2.3 & MSO \\
\hline 52444.900 & 0.199 & -47.4 & 1.0 & MSO \\
\hline 52505.874 & 0.261 & -46.9 & 0.5 & MSO \\
\hline 52630.234 & 0.387 & -47.3 & -0.2 & MSO \\
\hline 52686.819 & 0.444 & -46.0 & 1.5 & Gemini $\mathrm{S}$ \\
\hline 52749.616 & 0.508 & -47.1 & 1.2 & Gemini $\mathrm{S}$ \\
\hline 53129.768 & 0.894 & -56.3 & -0.6 & KPNO \\
\hline 53130.745 & 0.895 & -56.2 & -0.5 & KPNO \\
\hline 53131.761 & 0.896 & -57.8 & -2.1 & KPNO \\
\hline 53178.691 & 0.944 & -55.3 & 0.3 & KPNO \\
\hline 53493.787 & 0.263 & -47.6 & -0.2 & KPNO \\
\hline 53494.784 & 0.265 & -47.2 & 0.2 & KPNO \\
\hline 53537.651 & 0.308 & -45.5 & 1.6 & KPNO \\
\hline 53828.622 & 0.603 & -52.0 & -2.0 & Gemini S \\
\hline 53859.752 & 0.635 & -52.6 & -1.9 & KPNO \\
\hline 54230.718 & 0.012 & -53.9 & 0.3 & KPNO \\
\hline 54231.796 & 0.013 & -53.6 & 0.6 & KPNO \\
\hline 54270.690 & 0.052 & -52.6 & 0.3 & KPNO \\
\hline 54592.702 & 0.379 & -47.3 & -0.2 & KPNO \\
\hline 54634.666 & 0.422 & -49.0 & -1.7 & KPNO \\
\hline 54956.720 & 0.749 & -53.2 & 0.1 & KPNO \\
\hline 54975.515 & 0.768 & -52.3 & 1.4 & CTIO \\
\hline 54998.653 & 0.791 & -54.1 & 0.1 & KPNO \\
\hline 55270.894 & 0.068 & -54.4 & -2.1 & Gemini S \\
\hline 55310.615 & 0.108 & -49.6 & 1.3 & Gemini S \\
\hline 55320.732 & 0.118 & -50.0 & 0.6 & KPNO \\
\hline 55321.690 & 0.119 & -49.2 & 1.3 & KPNO \\
\hline 55362.691 & 0.161 & -49.7 & -0.4 & KPNO \\
\hline 55363.692 & 0.162 & -50.0 & -0.8 & KPNO \\
\hline 55693.734 & 0.497 & -48.4 & -0.2 & KPNO \\
\hline 55694.741 & 0.498 & -48.4 & -0.2 & KPNO \\
\hline 55727.653 & 0.531 & -49.0 & -0.3 & KPNO \\
\hline 56055.738 & 0.864 & -55.4 & 0.1 & KPNO \\
\hline 56099.684 & 0.909 & -54.7 & 1.1 & KPNO \\
\hline 56419.712 & 0.234 & -48.0 & -0.3 & KPNO \\
\hline 56420.766 & 0.235 & -48.0 & -0.3 & KPNO \\
\hline 56783.715 & 0.604 & -49.1 & 0.9 & KPNO \\
\hline 56825.656 & 0.646 & -50.3 & 0.6 & KPNO \\
\hline
\end{tabular}

Notes. MSO-Mount Stromlo Observatory, Gemini S-Gemini South Observatory, KPNO—Kitt Peak National Observatory, CTIO-Cerro Tololo Inter-American Observatory.

ellipsoidal variations (e.g., Mikołajewska 2001; Gromadzki et al. 2013). Thus, orbital periods may be determined from several types of light variability.

Gromadzki et al. (2013) carried out an extensive search for orbital and pulsational periods of symbiotic stars. From data taken in the huge photometric surveys of ASAS, MACHO, and OGLE, Gromadzki et al. (2013) analyzed the light curves of 79 symbiotic stars and determined probable orbital periods for 58 systems. Light curve residuals then were examined for pulsation periods.

Gromadzki et al. (2013) remarked in their Table 1 that in the ASAS observations V1044 Cen is blended with other stars in
Table 3

Orbital Elements and Related Parameters of V1044 Cen

\begin{tabular}{lc}
\hline \hline Parameter & Value \\
\hline$P($ days $)$ & $985.0 \pm 5.4$ \\
$T(\mathrm{HJD})$ & $2454219.2 \pm 51.8$ \\
$\gamma\left(\mathrm{km} \mathrm{s}^{-1}\right)$ & $-50.88 \pm 0.18$ \\
$K\left(\mathrm{~km} \mathrm{~s}^{-1}\right)$ & $4.39 \pm 0.25$ \\
$e$ & $0.158 \pm 0.057$ \\
$\omega($ degree $)$ & $224.0 \pm 20.1$ \\
$a$ sin $i\left(10^{6} \mathrm{~km}\right)$ & $58.7 \pm 3.3$ \\
$f(m)\left(M_{\odot}\right)$ & $0.0083 \pm 0.0014$ \\
Standard error of an observation of unit weight $\left(\mathrm{km} \mathrm{s}^{-1}\right)$ & 1.1 \\
\hline
\end{tabular}

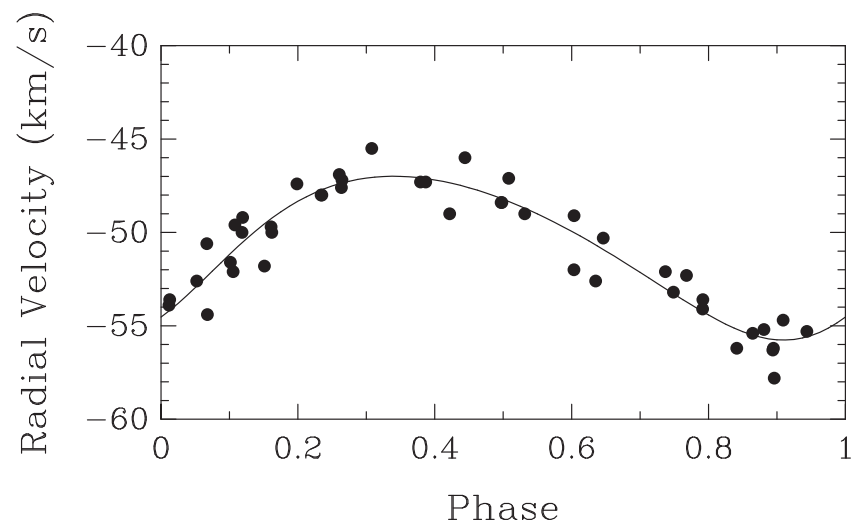

Figure 4. M giant radial velocities of V1044 Cen (filled circles) compared with its computed eccentric orbit (solid line). Zero phase is a time of periastron passage.

the field, and so its light curve was not analyzed because of contamination problems. While Gromadzki et al. (2013) did not examine its ASAS light curve, Wils (2003) did and estimated a period of 101: days.

As noted in Table 3, from the orbital solution of V1044 Cen the standard error of a radial velocity of unit weight is $1.1 \mathrm{~km} \mathrm{~s}^{-1}$. This value is twice as large as that found for several other symbiotic orbits in our series (e.g., Fekel et al. 2001). Thus, the velocity residuals of the adopted orbit were searched for periods between 10 and 500 days. The four best potential pulsation periods are 30.6, 35.0, 150.3, and 253.7 days, but none of these are similar to the possible value found by Wils (2003).

Using the extensive OGLE data base, Soszyński et al. (2007) determined period-luminosity relations for variable red giants in the Large and Small Magellanic Clouds. In the next section we determined an absolute magnitude, $M_{K}$, of -7.4 mag for V1044 Cen, which with the use of the coefficients for the O-rich semi-regular variables in the Large Magellanic Cloud and the adoption of $18.50 \mathrm{mag}$ for its distance modulus (Freedman et al. 2001), enables us to predict the fundamental and first overtone pulsation periods for the star. The predicted fundamental period is 234 days while the first overtone is 111 days. While our 253.7 days period is similar to that of the predicted fundamental period, the phase plot of the velocity distribution does not provide a particularly convincing case for its reality. 


\subsection{Discussion}

V1044 Cen has been part of several photometric and spectroscopic surveys. Swings \& Allen (1972) conducted an infrared survey of symbiotic and VV Cep stars to look for infrared excesses associated with circumstellar material and measured $H$ and $K$ magnitudes of V1044 Cen. Munari et al. (1992) made an optical and infrared photometric survey of symbiotic stars and obtained both $U B V R_{c} I_{c}$ and infrared $J H K$ observations of it. Their values for $V, K$, and $J-K$ are 11.15, 5.43 , and $1.28 \mathrm{mag}$, respectively. Gutierrez-Moreno et al. (1992) estimated a spectral class of M4.6, while from a more extensive spectrum analysis Mürset \& Schmid (1999) classified it as M5.5, which we adopt.

Schmutz et al. (1994), and Mürset et al. (2000), based on the theoretical work of Zahn (1977), have argued that the giant star in most S-type symbiotics is synchronously rotating. Making that assumption, they then went on to estimate the radius of the giant in various symbiotic binaries. In our analysis we adopt the synchronous rotation assumption. However, the orbit of V1044 Cen is not circular, but has a modest eccentricity of 0.16. According to Hut (1981), in such a situation the rotational angular velocity will tend to synchronize with that of the orbital motion at periastron, a condition he calls pseudosynchronous rotation. With Equation (42) of Hut (1981) we calculated a pseudosynchronous period of 853.4 days.

If the cool giant is pseudosynchronously rotating, then its projected rotational velocity can be used to estimate its minimum radius. For V1044 Cen we determined a $v \sin i$ value of $7.4 \pm 1.0 \mathrm{~km} \mathrm{~s}^{-1}$, which is in good agreement with the average value of $7.6 \pm 1.5 \mathrm{~km} \mathrm{~s}^{-1}$ from Zamanov et al. (2007). The minimum radius (i.e., $\sin i=1$ ) is $125 \pm 17 R_{\odot}$

From Table 3, for V1044 Cen the value of the mass function, which relates the primary and secondary masses and the orbital inclination (Batten et al. 1989), is quite small, just $0.008 M_{\odot}$. If we adopt typical masses of 1.5 and 0.5 (Mikołajewska 2003) for the $\mathrm{M}$ giant and its companion, then the orbital inclination is $40^{\circ}$. If as is generally assumed, the orbital and rotational axes are parallel, so the two inclinations are equal, the radius of the M giant would be increased from 125 to $194 R_{\odot}$.

The effective Roche lobe radius of the giant depends on the separation of the stars and their mass ratio. The adopted typical masses of 1.5 and $0.5 M_{\odot}$ (Mikołajewska 2003) for the M-giant and its companion, respectively, result in a mass ratio of 3.0. Next, we used Kepler's third law to determine the semimajor axis of the orbit. However, because the separation of the two components varies in an eccentric orbit, we adopted the smaller periastron separation rather than the semimajor axis. With Equation (2) of Eggleton (1983), we estimated a Roche-lobe radius of $210 R_{\odot}$ for the $\mathrm{M}$ giant at periastron. Our estimated radius of $192 R_{\odot}$ would fill $92 \%$ of that Roche lobe. This result is consistent with the conclusion of Mürset \& Schmid (1999), who found that symbiotics are, almost without exception, detached binaries. Such a large Roche lobe filling ratio should cause ellipsoidal light variations that would be most obvious at red or near-infrared wavelengths although the estimated inclination of $40^{\circ}$ would reduce the ellipsoidal amplitude.

From our adopted spectral class of M5.5 (Mürset \& Schmid 1999) we assume an effective temperature of $3425 \mathrm{~K}$ (Dyck et al. 1996). That, combined with our radius, results in a luminosity of $4640 L_{\odot}$, leading to $M_{\text {bol }}=-4.4$ mag. Including its error, the minimum radius of $108 R_{\odot}$ produces a luminosity of $1438 L_{\odot}$ and $M_{\mathrm{bol}}=-3.2 \mathrm{mag}$.

To estimate the distance to V1044 Cen, we adopted its $K$ mag and $J-K$ color from Munari et al. (1992). We then used the analytic expression of Bessell \& Wood (1984), involving that color, to obtain a bolometric correction at $K$. This, combined with our value of $M_{\mathrm{bol}}$ from the $\mathrm{M}$ giant's radius, produces $M_{K}=-7.4 \mathrm{mag}$ and results in a distance of $3.8 \mathrm{kpc}$ if reddening is ignored. V1044 Cen is situated about $52^{\circ}$ from the Galactic center and 25:6 above the Galactic plane, so it may not have a large amount of reddening at infrared wavelengths. If we nevertheless include an extinction $A_{K}$ value of $0.3 \mathrm{mag}$, the same as that estimated for Hen 3-1213 (Gutierrez-Moreno et al. 1999), the distance decreases to $3.3 \mathrm{kpc}$. If the minimum radius of $108 R_{\odot}$ is adopted, then $M_{K}=-6.2 \mathrm{mag}$ and if no reddening is assumed, a distance of $2.1 \mathrm{kpc}$ results. The above extinction value reduces the distance to $1.8 \mathrm{kpc}$. Thus, depending on the adopted stellar radius and amount of extinction, the distance to V1044 Cen ranges from 1.8 to $3.8 \mathrm{kpc}$.

\section{HEN 3-1213 = SS 73-60 \\ 7.1. Short History}

Wackerling (1970) noted that Hen 3-1213 is a symbiotic star in his extensive catalog of early-type stars whose spectra have shown emission lines. This identification was based on a spectrum acquired by Carlson (1968) that showed an M star continuum and He II in emission (see Henize 1976). Sanduleak \& Stephenson (1973) carried out an objective prism survey of the southern Milky Way, looking for stars with strong emission lines. In their 1973 list they assigned it number 60 and characterized it as a $\mathrm{Z}$ Andromedae like star having a $\mathrm{He}$ II $4686 \AA$ A emission feature and a continuum of spectral type M2. Several years later, Henize (1976) published an extensive list of results (now called the third catalog) from the MichiganMount Wilson southern $\mathrm{H} \alpha$ survey. The same star was featured as number 1213, the results of the spectrum by Carlson (1968) were mentioned, and the star was identified as a symbiotic system. Allen (1978) obtained a low dispersion slit spectrum of Hen 3-1213 that confirmed its identity as a symbiotic. He described the continuum as being strong, the Balmer decrement as shallow, and emission lines of $\mathrm{He}$ I, He II, plus several forbidden emission features in its spectrum. From a $2 \mu \mathrm{m}$ spectrum taken with the Anglo-Australian Telescope Allen (1980) determined a spectral class of K4. In his catalog of symbiotic stars Allen (1984) provided a plot of its spectrum with a wavelength range of 3400-7500 A. Gutierrez-Moreno et al. (1999) determined its Johnson $B$ and $V$ magnitudes and obtained low-dispersion optical spectra from which they estimated a spectral class of M0.0. Along with over 90 other symbiotics Mürset \& Schmid (1999) classified the spectrum of Hen 3-1213 based on near-infrared spectra. They found no TiO bands visible and gave it a spectral class of $\leqslant \mathrm{K} 4$, consistent with the result of Allen (1980). Such a spectral class indicates that it is one of a small group of stars called yellow symbiotics (Allen et al. 1982; Schmid \& Nussbaumer 1993; Pereira \& Roig 2009). Pereira \& Roig (2009) acquired high-resolution optical spectra and determined its chemical composition. They found that Hen 3-1213 is a metal-poor K3 giant, enriched in s-process elements and with an iron abundance $[\mathrm{Fe} / \mathrm{H}]=-0.93$. Thus, they concluded that it belongs to the halo population. 
Table 4

Absorption Line Radial Velocities of Hen 3-1213

\begin{tabular}{lccrl}
\hline \hline $\begin{array}{l}\text { HJD } \\
-2400000\end{array}$ & Phase & $\begin{array}{l}\text { Velocity } \\
\left(\mathrm{km} \mathrm{s}^{-1}\right)\end{array}$ & $\begin{array}{r}O-C \\
\left(\mathrm{~km} \mathrm{~s}^{-1}\right)\end{array}$ & Observatory \\
\hline 51993.240 & 0.045 & -41.4 & -0.6 & MSO \\
52046.104 & 0.144 & -44.9 & 0.9 & MSO \\
52094.100 & 0.234 & -47.1 & 0.4 & MSO \\
52131.002 & 0.303 & -47.9 & -0.5 & MSO \\
52155.879 & 0.350 & -47.4 & -0.5 & MSO \\
52351.265 & 0.717 & -37.2 & -0.1 & MSO \\
52399.110 & 0.806 & -34.6 & 0.2 & MSO \\
52445.127 & 0.893 & -34.7 & -0.4 & MSO \\
52504.896 & 0.005 & -37.6 & 0.9 & MSO \\
52505.937 & 0.007 & -38.6 & -0.0 & MSO \\
52686.859 & 0.346 & -46.3 & 0.6 & Gemini S \\
52749.742 & 0.464 & -44.9 & -0.3 & Gemini S \\
52866.486 & 0.683 & -37.3 & 0.8 & Gemini S \\
53098.691 & 0.119 & -45.8 & -1.0 & Gemini S \\
54969.823 & 0.630 & -39.9 & -0.1 & CTIO \\
55036.628 & 0.755 & -36.5 & -0.5 & CTIO \\
55320.876 & 0.288 & -47.6 & -0.1 & CTIO \\
55340.587 & 0.325 & -47.0 & 0.2 & Gemini S \\
\hline
\end{tabular}

Notes. MSO-Mount Stromlo Observatory, Gemini S-Gemini South Observatory, CTIO — Cerro Tololo Inter-American Observatory.

Gromadzki et al. (2013) examined the ASAS database (Pojmanski 2002) light curve for Hen 3-1213 and found a period of 514 days that they associated with the orbital period. They also examined the light curve residuals and identified a period of 191 days, which they ascribed to pulsation.

\subsection{Spectroscopic Orbit}

Between 2001 March and 2010 May we acquired 18 spectra of Hen 3-1213 (Table 4). An analysis of these velocities resulted in a preliminary period of 536 days. Our initial orbitalelement solution indicated that the orbit is eccentric, and our final orbit elements, which include a period of $533.0 \pm 2.0$ days and an eccentricity of $0.18 \pm 0.03$, are presented in Table 5 . For this orbit the rms of an individual radial velocity is just $0.5 \mathrm{~km} \mathrm{~s}^{-1}$, which is a very low value for the symbiotic orbits in our series. This indicates that pulsation is not a significant contributor to the radial velocities. A phase plot of the radial velocities compared to the computed velocity curve is shown in Figure 5.

\subsection{Discussion}

From a spectroscopic analysis Pereira \& Roig (2009) determined an effective temperature of $4100 \mathrm{~K}$ corresponding to a K3 spectral class for Hen 3-1213. Schmid \& Nussbaumer (1993) applied the term "yellow symbiotics" to symbiotic binaries that have temperatures between 4000 and $7000 \mathrm{~K}$, which make them warmer than traditional M-giant symbiotics. Thus, according to their definition, Hen 3-1213 is a member of that small subsample of symbiotics.

Like V1044 Cen the orbit of Hen 3-1213 is not circular but has an eccentricity of 0.18 . Thus, with Equation (42) of Hut (1981) we computed a pseudosynchronous period of 445.8 days. From two methods, Zamanov et al. (2007) obtained an average projected rotational velocity of $10 \pm$ $1.5 \mathrm{~km} \mathrm{~s}^{-1}$. From our spectra we find a somewhat smaller value for $v \sin i$ of $7.0 \pm 1.0 \mathrm{~km} \mathrm{~s}^{-1}$. Our value and the assumption of
Table 5

Orbital Elements and Related Parameters of Hen 3-1213

\begin{tabular}{lc}
\hline \hline Parameter & Value \\
\hline$P($ days $)$ & $533.0 \pm 2.0$ \\
$T(\mathrm{HJD})$ & $2453568.2 \pm 18.1$ \\
$\gamma\left(\mathrm{km} \mathrm{s}^{-1}\right)$ & $-41.41 \pm 0.19$ \\
$K\left(\mathrm{~km} \mathrm{~s}^{-1}\right)$ & $6.69 \pm 0.25$ \\
$e$ & $0.183 \pm 0.034$ \\
$\omega($ degree $)$ & $65.9 \pm 13.0$ \\
$a$ sin $i\left(10^{6} \mathrm{~km}\right)$ & $48.2 \pm 1.8$ \\
$f(m)\left(M_{\odot}\right)$ & $0.0157 \pm 0.0018$ \\
Standard error of an observation of unit weight $\left(\mathrm{km} \mathrm{s}^{-1}\right)$ & 0.65 \\
\hline
\end{tabular}

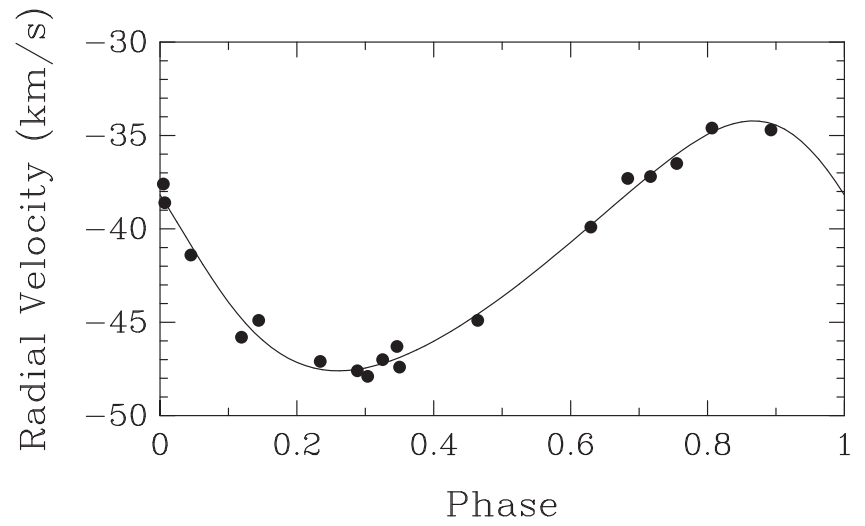

Figure 5. K giant radial velocities of Hen 3-1213 (filled circles) compared with its computed orbit (solid line). Zero phase is a time of periastron passage.

pseudosynchronous rotation produce a minimum radius of 62 $\pm 9 R_{\odot}$ for Hen 3-1213.

Pereira \& Roig (2009) found Hen 3-1213 to be metal-poor with $[\mathrm{Fe} / \mathrm{H}]=-0.93$ and concluded that the $\mathrm{K}$ giant belongs to the halo population. Smith et al. (1997) noted that halo giants are believed to have masses ranging from about 0.8 to $1.0 M_{\odot}$. Thus, we adopt a mass of $1.0 M_{\odot}$ for the $\mathrm{K}$ giant primary and $0.5 M_{\odot}$ for its putative white dwarf companion.

Like that of V1044 Cen the mass function value of Hen 31213 is relatively small, $0.016 M_{\odot}$, and so the orbital inclination is not expected to be close to $90^{\circ}$. Adopting the above masses results in an orbital inclination of $41^{\circ}$. If the orbital and rotational inclinations are equal, as is usually assumed, then the $\mathrm{K}$ giant radius increases from $62 \pm 9 R_{\odot}$ to $94 R_{\odot}$. The Roche lobe radius at periastron is $114 R_{\odot}$, so under the above assumptions, the $\mathrm{K}$ giant fills $82 \%$ of its Roche lobe at that time. Thus, the system appears to be detached in agreement with the conclusion of Mürset \& Schmid (1999). Once again, the substantial filling of the Roche lobe indicates that ellipsoidal variations could be found at red or infrared wavelengths although their amplitude would be decreased because of the low estimated inclination of $40^{\circ}$. However, we note that Gromadzki et al. (2013) presented a $V$ light curve for Hen 3-1213 that showed a relatively deep minimum of about 0.3-0.4 mag, which suggests a higher inclination.

The above effective temperature and radius produce $M_{\text {bol }}=-3.6$ mag. The biggest uncertainty in the computation is the radius. If we adopt the minimum radius of $62 R_{\odot}$ minus its error of $9 R_{\odot}$, then $M_{\text {bol }}$ is reduced to $-2.4 \mathrm{mag}$. With the $K$ and $J-K$ values given by Gutierrez-Moreno et al. (1999) and the bolometric correction computed from the relation of Bessell $\&$ Wood (1984), for a radius of $94 R_{\odot}$ we determine 
$M_{K}=-6.7 \mathrm{mag}$, which leads to a distance of $4.9 \mathrm{kpc}$ if reddening is ignored. Including an extinction value, $A_{K}$, of 0.3 mag (Gutierrez-Moreno et al. 1999) reduces the distance to $4.3 \mathrm{kpc}$. Adopting a much smaller value of $53 R_{\odot}$, the minimum radius reduced by its error, we determine $M_{K}=-5.5 \mathrm{mag}$. Without extinction this results in a distance of $2.8 \mathrm{kpc}$, and with the above extinction value is reduced to $2.4 \mathrm{kpc}$, which is still somewhat larger than the value of $1.7 \mathrm{kpc}$ that was estimated by Pereira \& Roig (2009). To come close to that value requires reducing the radius of the $\mathrm{K}$ giant by half, $47 R_{\odot}$, and including the extinction, which then results in a distance of $2.1 \mathrm{kpc}$. We note that the position of Hen 3-1213 in the sky is close to the Galactic center, being just $26^{\circ}$ away in longitude and only $3^{\circ}$ below the Galactic plane.

\section{SS 73-96 \\ 8.1. Short History}

From an objective prism survey of strong emission line objects in the southern Milky Way, Sanduleak \& Stephenson (1973) listed the star as number 96 and assigned it to a category that they called Be! pec. Such objects have very strong hydrogen emission lines, and in the case of SS 73-96, He I emission at $5876 \AA$ was also suspected to be present. Allen (1978) obtained low dispersion slit spectra of a number of objects in the list of Sanduleak \& Stephenson (1973). He found SS 73-96 to have "clear TiO bands in a faint continuum" and many forbidden emission lines including [Fe VI] and [ $\mathrm{Fe} \mathrm{VII}]$. He concluded that the star was a symbiotic. Allen (1980) acquired a $2 \mu \mathrm{m}$ spectrum from which he classified SS 73-96 as M2. Munari \& Patat (1993) searched for an $\mathrm{H} \alpha$ nebula around the star but did not find one.

\subsection{Spectroscopic Orbit}

From 2001 May to 2014 June we obtained 33 radial velocities of SS 73-96 (Table 6). An analysis of these velocities, produced an initial period of 827 days. Adopting this period, we determined preliminary orbital elements, which were then refined and produced a period of $828.2 \pm 2.0$ days. The orbit is clearly eccentric with $e=0.26 \pm 0.03$. Our orbital elements are given in Table 7 and the radial velocities are compared with the computed orbit in Figure 6.

\subsection{Emission Line Spectrum}

Mikołajewska et al. (1989) stated that the He II emission should follow the orbit of the hot component. Additional analyses by Quiroga et al. (2002) and Brandi et al. (2005), who measured a variety of emission lines in the 4000-7300 $\AA$ range, found that the wings of the $\mathrm{H} \alpha, \mathrm{H} \beta$, and $\mathrm{He}$ II lines do seem to follow the hot component. The $\mathrm{H} \alpha$ profiles of several symbiotics, including FG Ser, AR Pav, and FN Sgr, show a double peaked emission feature that has central absorption (Mürset et al. 2000; Quiroga et al. 2002; Brandi et al. 2005). Attempts to model the emission (e.g., Robinson et al. 1994) have been mostly unsatisfactory, and there is no agreement yet as to whether the $\mathrm{H} \alpha$ emission comes from an accretion disk around the white dwarf companion or results from an extended wind from the giant (Gorlova et al. 2012).

Having determined our orbit for the $\mathrm{M}$ giant, we examined our 22 KPNO spectra of the $1 \mu \mathrm{m}$ region, which have both $\mathrm{H}_{\mathrm{I}}$ Paschen $\delta$ and He II emission lines. Neither show the very
Table 6

Absorption Line Radial Velocities of SS 73-96

\begin{tabular}{|c|c|c|c|c|}
\hline $\begin{array}{l}\text { HJD } \\
-2400000\end{array}$ & Phase & $\begin{array}{l}\text { Velocity } \\
\left(\mathrm{km} \mathrm{s}^{-1}\right)\end{array}$ & $\begin{array}{r}O-C \\
\left(\mathrm{~km} \mathrm{~s}^{-1}\right)\end{array}$ & Observatory \\
\hline 52047.035 & 0.930 & -54.2 & -0.7 & MSO \\
\hline 52095.202 & 0.988 & -56.4 & -0.7 & MSO \\
\hline 52132.165 & 0.033 & -55.2 & 0.0 & MSO \\
\hline 52353.308 & 0.300 & -41.2 & -0.4 & MSO \\
\hline 52400.238 & 0.356 & -41.3 & -2.0 & MSO \\
\hline 52447.090 & 0.413 & -39.2 & -0.8 & MSO \\
\hline 52507.066 & 0.485 & -37.6 & 0.4 & MSO \\
\hline 52749.942 & 0.779 & -43.9 & 0.2 & Gemini S \\
\hline 53098.859 & 0.200 & -44.4 & 0.6 & Gemini S \\
\hline 53131.921 & 0.240 & -42.6 & 0.4 & KPNO \\
\hline 53178.894 & 0.296 & -40.7 & 0.2 & KPNO \\
\hline 53493.943 & 0.677 & -39.0 & 1.2 & KPNO \\
\hline 53538.881 & 0.731 & -41.7 & 0.3 & KPNO \\
\hline 53899.854 & 0.167 & -47.0 & -0.1 & KPNO \\
\hline 54231.910 & 0.568 & -39.7 & -1.4 & KPNO \\
\hline 54271.839 & 0.616 & -39.6 & -0.7 & KPNO \\
\hline 54634.809 & 0.054 & -53.8 & 0.4 & KPNO \\
\hline 54960.941 & 0.448 & -37.1 & 1.0 & KPNO \\
\hline 54999.816 & 0.495 & -36.1 & 1.9 & KPNO \\
\hline 55037.746 & 0.541 & -37.1 & 1.0 & CTIO \\
\hline 55320.940 & 0.883 & -50.7 & -0.3 & KPNO \\
\hline 55324.898 & 0.888 & -51.6 & -0.9 & KPNO \\
\hline 55349.668 & 0.918 & -51.6 & 1.1 & Gemini S \\
\hline 55362.823 & 0.933 & -53.5 & 0.2 & KPNO \\
\hline 55363.826 & 0.935 & -53.1 & 0.7 & KPNO \\
\hline 55693.936 & 0.333 & -39.4 & 0.4 & KPNO \\
\hline 55728.810 & 0.375 & -38.5 & 0.5 & KPNO \\
\hline 56055.889 & 0.770 & -42.7 & 1.0 & KPNO \\
\hline 56099.812 & 0.823 & -46.5 & 0.0 & KPNO \\
\hline 56419.911 & 0.210 & -45.1 & -0.6 & KPNO \\
\hline 56461.817 & 0.260 & -42.2 & -0.1 & KPNO \\
\hline 56785.907 & 0.652 & -41.2 & -1.6 & KPNO \\
\hline 56825.807 & 0.700 & -42.0 & -1.1 & KPNO \\
\hline
\end{tabular}

Notes. MSO-Mount Stromlo Observatory, Gemini S-Gemini South Observatory, KPNO = Kitt Peak National Observatory, CTIO-Cerro Tololo Inter-American Observatory.

Table 7

Orbital Elements and Related Parameters of SS 73-96

\begin{tabular}{lcc}
\hline \hline Parameter & M Giant & H I \\
& Absorption & Emission \\
\hline$P$ (days) & $828.2 \pm 2.0$ & 828.2 (fixed) \\
$T($ HJD $)$ & 2454589.8 & 2454713.8 \\
& \pm 13.1 & \pm 56.0 \\
$\gamma\left(\mathrm{km} \mathrm{s}^{-1}\right)$ & $-44.58 \pm 0.18$ & $-39.2 \pm 1.4$ \\
$K\left(\mathrm{~km} \mathrm{~s}^{-1}\right)$ & $8.89 \pm 0.27$ & $21.6 \pm 2.0$ \\
$e$ & $0.256 \pm 0.028$ & $0.190 \pm 0.095$ \\
$\omega($ degree $)$ & $180.6 \pm 6.0$ & $87.1 \pm 25.1$ \\
$a$ sin $i\left(10^{6} \mathrm{~km}\right)$ & $97.9 \pm 3.1$ & $241.4 \pm 22.6$ \\
$f(m)\left(M_{\odot}\right)$ & $0.0547 \pm 0.0051$ & $0.82 \pm 0.23$ \\
Standard error of an observation of & 0.93 & 5.5 \\
$\quad$ unit weight $\left(\mathrm{km} \mathrm{s}^{-1}\right)$ & & \\
\hline
\end{tabular}

strong wings that are seen in the profiles of $\mathrm{H} \alpha$ and to a lesser extent the He II $4686 \AA$ line (Quiroga et al. 2002; Brandi et al. 2005). However, many of the Paschen $\delta$ emission profiles of SS 73-96 have well defined, double peaked structure, resembling profiles seen for $\mathrm{H} \alpha$ in other symbiotics as noted previously. At times this makes it more difficult for us to 


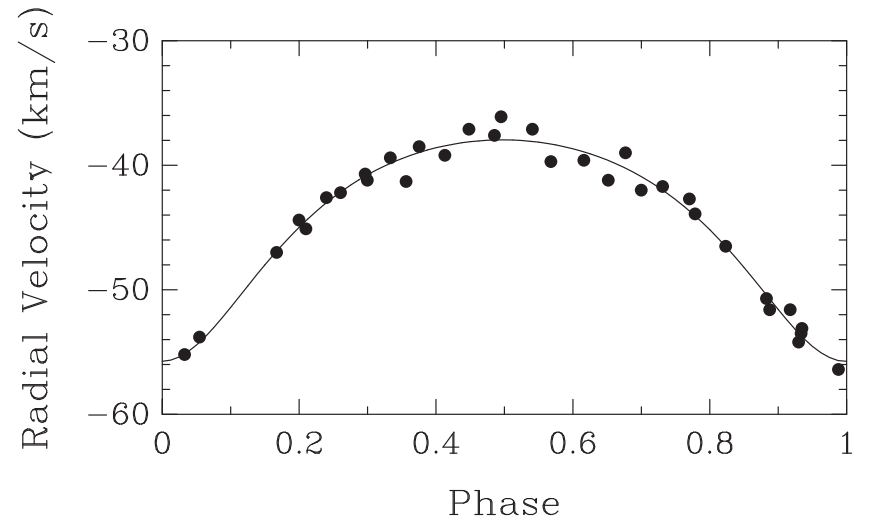

Figure 6. M giant radial velocities of SS 73-96 (filled circles) compared with its computed orbit (solid line). Zero phase is a time of periastron passage.

measure the emission lines, and our fits involve the whole profile. In all but one of our 22 spectra the He II emission line is stronger than the Paschen $\delta$ feature.

In Table 8 we list the phase of the $M$ giant orbit and the velocities of the two emission lines. Figure 7 then compares the $M$ giant orbit with the radial velocities of the two emission features. As was found for AE Ara (Fekel et al. 2010), the Paschen $\delta$ feature appears to provide the more likely orbit. A forced-fit curve of those velocities with their orbit $180^{\circ}$ out of phase with the $\mathrm{M}$ giant orbit is shown. That curve results in a semiamplitude of $17.5 \pm 5.0 \mathrm{~km} \mathrm{~s}^{-1}$ for the emission line velocities and produces a primary to secondary mass ratio of $2.0 \pm 0.5$.

Compared to the forced-fit curve, the emission velocities are clearly systematically shifted. Thus, we adopted the orbital period determined from the $M$ giant velocities and then obtained an orbit for the Paschen $\delta$ emission velocities with all other elements varied. That emission line solution is listed in Table 7. The semiamplitude of the emission line velocities from that orbit produces a mass ratio of $2.4 \pm 0.2$. For 16 systems Mikołajewska (2003) estimated symbiotic system mass ratios that ranged from 2 to 4, so our result for SS 73-96 is consistent with that range.

\subsection{Pulsation Period Search}

We examined the velocity residuals to the adopted eccentric orbit by searching for periods between 10 and 500 days. The best two possible periods were 44.2 and 315.1 days. SS 73-96 was not included in the period search survey of Gromadzki et al. (2013). However, we note that of the 33 periods that Gromadzki et al. (2013) ascribed to pulsation only MWC 560 with a period of 332 days is not between 40 and 200 days, a period range very similar to the typical pulsation range noted by Lebzelter \& Hinkle (2002). The phase diagram for our possible 44.2 days period is not convincing and although that for the 315.1 days period looks reasonable, such a period is significantly longer than that found for nearly all other pulsating symbiotics. In addition, as we did for V1044 Cen, we predict the pulsation periods of SS 73-96 using its $M_{K}$ of $-6.6 \mathrm{mag}$ and the period-luminosity relationship of Soszyński et al. (2007). We find a fundamental period of 143 days and a first overtone period of 69 days. The only period in our velocity residual data that is close to the fundamental period is 169.2 days, and its phase diagram is shown in Figure 8. The ratio of the full amplitude of the velocity variation to its
Table 8

Emission Line Radial Velocities of SS 73-96

\begin{tabular}{|c|c|c|c|}
\hline $\begin{array}{l}\text { HJD- } \\
2400000\end{array}$ & Phase & $\begin{array}{c}\text { H I } \\
\text { Velocity } \\
\left(\mathrm{km} \mathrm{s}^{-1}\right)\end{array}$ & $\begin{array}{c}\text { He II } \\
\text { Velocity } \\
\left(\mathrm{km} \mathrm{s}^{-1}\right)\end{array}$ \\
\hline 53131.921 & 0.240 & -55.5 & -70.1 \\
\hline 53178.894 & 0.296 & -58.2 & -66.9 \\
\hline 53493.943 & 0.677 & -29.3 & -42.3 \\
\hline 53538.881 & 0.731 & -31.3 & -36.5 \\
\hline 53899.854 & 0.167 & -42.4 & -76.3 \\
\hline 54231.910 & 0.568 & -57.7 & -54.0 \\
\hline 54271.839 & 0.616 & -36.6 & -49.9 \\
\hline 54634.809 & 0.054 & -20.8 & -45.8 \\
\hline 54960.941 & 0.448 & -62.4 & -67.8 \\
\hline 54999.816 & $0.495^{\mathrm{a}}$ & $\ldots$ & -57.9 \\
\hline 55320.940 & 0.883 & -25.6 & -28.4 \\
\hline 55324.898 & 0.888 & -24.7 & -26.8 \\
\hline 55362.823 & 0.933 & -16.9 & -16.5 \\
\hline 55363.826 & 0.935 & -16.2 & -16.8 \\
\hline 55693.936 & 0.333 & -57.6 & -63.5 \\
\hline 55728.810 & 0.375 & -53.2 & -62.2 \\
\hline 56055.889 & 0.770 & -23.7 & -34.4 \\
\hline 56099.812 & 0.823 & -23.8 & -27.6 \\
\hline 56419.911 & 0.210 & -45.6 & -74.3 \\
\hline 56461.817 & 0.260 & -62.4 & -71.2 \\
\hline 56785.907 & 0.652 & -41.1 & -51.2 \\
\hline 56825.807 & 0.700 & -43.8 & -51.8 \\
\hline
\end{tabular}

Note.

${ }^{\mathrm{a}}$ The Paschen $\delta$ line is very weak and poorly defined at this phase.

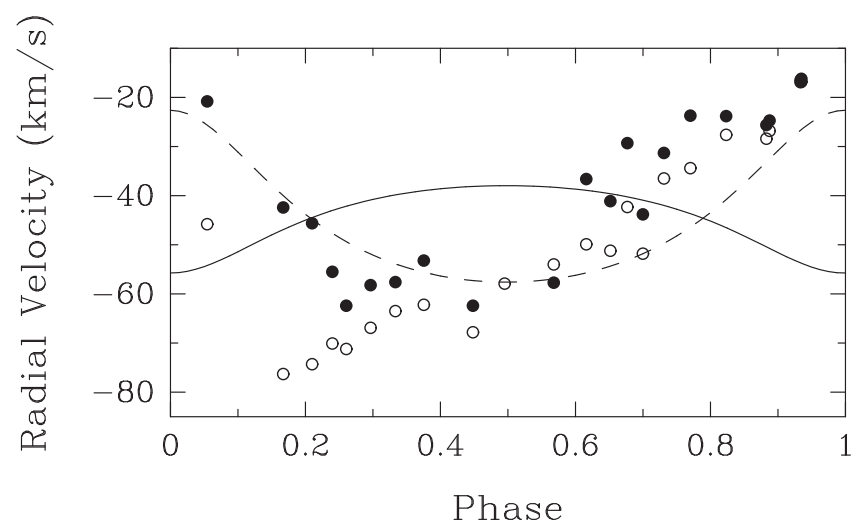

Figure 7. Emission line radial velocities of SS 73-96 (filled circles $=\mathrm{H}$ I, open circles $=\mathrm{He}$ II) compared to the orbit determined for the M giant (solid line) and the forced fit curve for the $\mathrm{H}$ i emission lines (dashed line), which requires their orbit to be $180^{\circ}$ out of phase with the $\mathrm{M}$ giant orbit. Zero phase is a time of periastron passage.

uncertainty is 2.0 in each of our three possible periods. Thus, we conclude that it is unlikely that these periods are real.

\subsection{Discussion}

Basic photometric and spectroscopic observations are available for SS 73-96. Munari et al. (1992) made an optical and infrared photometric survey of symbiotic stars and obtained both $U B V R_{c} I_{c}$ and $J H K$ observations of SS 73-96. They determined $V, K$, and $J-K$ values of $15.24,6.36$, and $1.78 \mathrm{mag}$, respectively. Allen (1980) estimated a spectral class of $\mathrm{M} 2$ from a $2 \mu \mathrm{m}$ spectrum.

Computed from the orbital elements of SS 73-96, the value of $0.055 M_{\odot}$ for the mass function of the primary (Table 7 ) is 


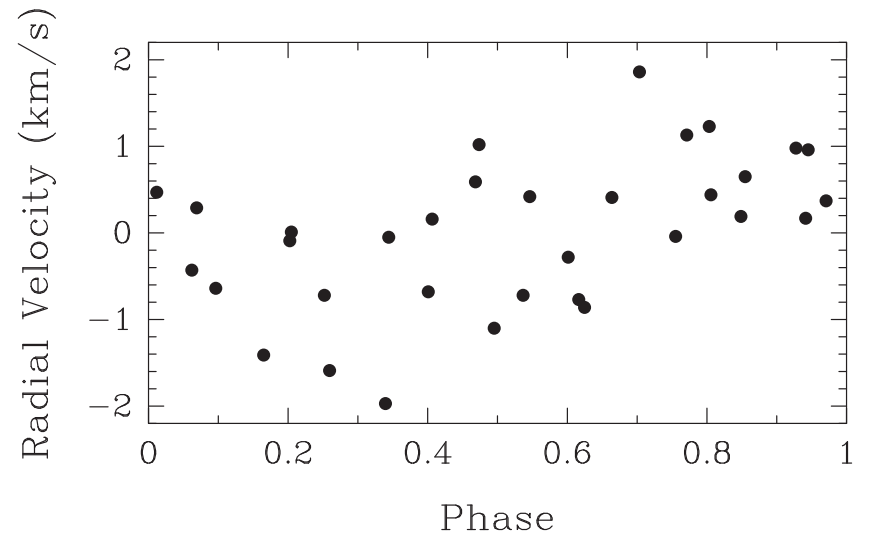

Figure 8. M giant residual radial velocities of SS 73-96 plotted with a period of 169.2 days and zero phase epoch of JD 2455050 . The residual velocity phase diagrams for periods of 44.2 and 315.1 days have similar amplitudes.

relatively large for symbiotic systems, suggesting that the system might have eclipses. If we adopt a typical mass of $1.5 M_{\odot}$ (Mikołajewska 2003) for the $\mathrm{M}$ giant, the mass ratio of 2.4 produces $0.625 M_{\odot}$ for its companion. Substituting those values into the mass function equation for the $\mathrm{M}$ giant results in an orbital inclination of $90^{\circ}$.

From our spectra our $v \sin i$ measurement is $8.0 \pm$ $1.0 \mathrm{~km} \mathrm{~s}^{-1}$. With that value, the assumption of pseudosynchronous rotation, and a pseudosynchronous period of 591.4 days, the minimum radius of $\mathrm{SS} 73-96$ is $94 \pm 12 R_{\odot}$. If as is generally assumed, the orbital and rotational axes are parallel, then $\sin i$ is 1 , and the radius of the $\mathrm{M}$ giant is $94 R_{\odot}$. The Roche lobe radius at periastron is $162 R_{\odot}$, so the $\mathrm{M}$ giant radius fills only $58 \%$ of its Roche lobe at periastron, consistent with the general conclusion of Mürset \& Schmid (1999) that symbiotic binaries are detached systems.

The M2 giant classification of Allen (1980), corresponds to an effective temperature of $3740 \mathrm{~K}$ (Dyck et al. 1996). That temperature and the above radius produce $M_{\mathrm{bol}}=-3.2 \pm$ 0.4 mag. With the $K$ and $J-K$ values given by Munari et al. (1992) and the bolometric correction computed from the relation of Bessell \& Wood (1984), we determine $M_{K}=-6.6$ $\pm 0.4 \mathrm{mag}$, which leads to a distance of $3.7 \pm 0.8 \mathrm{kpc}$ if reddening is ignored. SS 73-96 is just $7^{\circ}$ in longitude from the Galactic center and only $3^{\circ}$ below the Galactic plane. Thus, even at infrared wavelengths there may be significant extinction. A value for $A_{K}$ of $0.5 \mathrm{mag}$ reduces the distance to $3 \mathrm{kpc}$.

From our orbital solution in Table 7 the ephemeris for conjunctions with the $\mathrm{M}$ giant in front, which corresponds to a time of mid-eclipse, is

$$
T_{\text {conj }}(\mathrm{HJD})=2454728.8( \pm 13.1)+828.2( \pm 2.0) E,
$$

where $E$ is an integer number of cycles.

An eclipse of a supposed white dwarf component would produce minimal change in the overall visual light level. However, an eclipse of the extended hot disk surrounding the secondary (e.g., Hutchings et al. 1983) would be easily observed at optical wavelengths.

We appreciate the referee's helpful suggestions. We thank NOAO for enabling several aspects of this research, including support of the KPNO coudé feed telescope, access to time on the Gemini and SMARTS telescopes, and travel support for K.
Hinkle and R. Joyce. This paper is based in part on observations obtained at the Gemini Observatory, which is operated by the Association of Universities for Research in Astronomy, Inc., under a cooperative agreement with the NSF on behalf of the Gemini partnership: the National Science Foundation (United States), the Particle Physics and Astronomy Research Council (United Kingdom), the National Research Council (Canada), CONICYT (Chile), the Australian Research Council (Australia), CNPq (Brazil), and CONICRT (Argentina). The observations were acquired with the Phoenix infrared spectrograph, which was developed and is operated by the National Optical Astronomy Observatory. The Gemini/ Phoenix spectra were obtained as part of programs GS-2003ADD-1, GS-2003B-DD-1, GS-2004A-DD-1, and GS-2006ADD-1. Observations were also acquired at the Gemini South Observatory as part of Poor Weather Queue program number GS-2010A-Q-79. Several additional observations were acquired through the NOAO share of time on one of the SMARTS consortium telescopes. This research has been supported in part by NASA grants NCC5-511 and NSF grant HRD-9706268 to Tennessee State University. We have made use of the SIMBAD database, operated by CDS in Strasbourg, France, as well as NASA's Astrophysics Data System Abstract Service.

\section{REFERENCES}

Allen, D. A. 1978, MNRAS, 184, 601

Allen, D. A. 1980, MNRAS, 192, 521

Allen, D. A. 1982, in IAU Coll. 70, The Nature of Symbiotic Stars, ed. M. Friedjung \& R. Viotti (Dordretch: Reidel), 27

Allen, D. A. 1984, PASAu, 5, 369

Batten, A. H., Fletcher, J. M., \& MacCarthy, D. G. 1989, PDAO, 17, 1

BelczyŃski, K., Mikołajewska, J., Munari, U., Ivison, R. J., \& Friedjung, M. 2000, A\&AS, 146, 407

Bessell, M. S., \& Wood, P. R. 1984, PASP, 96, 247

Bidelman, W. P., \& MacConnell, D. J. 1973, AJ, 78, 687

Brandi, E., Mikołajewska, J., Quiroga, C., et al. 2005, A\&A, 440, 239

Carlson, E. D. 1968, PhD dissertation, Northwestern Univ.

Dobrzycka, D., Kenyon, S. J., \& Mikołjewska, J. 1993, AJ, 106, 284

Dyck, H. M., Benson, J. A., van Belle, G. T., \& Ridgway, S. T. 1996, AJ, 111,1705

Eggleton, P. P. 1983, ApJ, 269, 368

Fekel, F. C., Hinkle, K. H., \& Joyce, R. R. 2003, in ASP Conf. Ser. 303 Symbiotic Stars Probing Stellar Evolution, ed. R. L. M. Corradi, J. Mikołajewska \& T. J. Mahoney (San Francisco, CA: ASP), 113

Fekel, F. C., Hinkle, K. H., Joyce, R. R., \& Skrutskie, M. F. 2001, AJ, 121,2219

Fekel, F. C., Hinkle, K. H., Joyce, R. R., \& Wood, P. R. 2010, AJ, 139, 1315

Fekel, F. C., Hinkle, K. H., Joyce, R. R., Wood, P. R., \& Howarth, I. D. 2008, AJ, 136, 146

Fekel, F. C., Joyce, R. R., Hinkle, K. H., \& Skrutskie, M. F. 2000, AJ, 119,1375

Fitzpatrick, M. J. 1993, ASP Conf. Ser. 52, Astronomical Data Analysis Software and Systems II, ed. R. J. Hanisch, R. V. J. Brissenden \& J. Barnes (San Francisco, CA: ASP), 472

Freedman, W. L., Madore, B. F., \& Gibson, B. K. 2001, ApJ, 553, 47

Gorlova, N., van Winckel, H., \& Jorissen, A. 2012, BaltA, 21, 165

Gromadzki, M., Mikołajewska, J., \& Soszynski, I. 2013, AcA, 63, 405

Gutierrez-Moreno, A., Moreno, H., \& Cortes, G. 1992, PASP, 104, 1187

Gutierrez-Moreno, A., Moreno, H., \& Costa, E. 1999, PASP, 111, 571

Henize, K. 1976, ApJS, 30, 491

Hinkle, K. H., Cuberly, R. W., Gaughan, N. A., et al. 1998, Proc. SPIE, 3354, 810

Hinkle, K. H., Fekel, F. C., Joyce, R. R., \& Wood, P. R. 2013, ApJ, 770, 28 Hinkle, K. H., Fekel, F. C., Joyce, R. R., et al. 2006, AJ, 641, 479

Hut, P. 1981, A\&A, 99, 126

Hutchings, J. B., Cowley, A. P., Ake, T. B., \& Imhoff, C. L. 1983, ApJ, 275,271

Jorissen, A., van Eck, S., Dermine, T., van Winckel, H., \& Gorlova, N. 2012, BaltA, 21, 39 
Joyce, R. R. 1992, ASP Conf. Ser. 23, Astronomical CCD Observing and Reduction Techniques, ed. S. B. Howell (San Francisco, CA: ASP), 258

Joyce, R. R., Hinkle, K. H., Meyer, M. R., \& Skrutskie, M. F. 1998, Proc SPIE, 3354, 741

Kazarovets, E. V., Kireeva, N. N., Samus, N. N., \& Durlevich, O. V. 2003, IBVS, 5422, 1

Keenan, P. C., \& McNeil, R. C. 1989, ApJS, 71, 245

Lebzelter, T., \& Hinkle, K. H. 2002, A\&A, 393, 563

Lucy, L. B., \& Sweeney, M. A. 1971, AJ, 76, 544

Mikołajewska, J. 2001, in IAU Coll. 183, Small Telescope Astronomy on Global Scales, ed. W.-P. Chen, C. Lemme \& B. Paczyński (San Francisco, CA: ASP), 167

Mikołajewska, J. 2003, in Symbiotic Stars Probing Stellar Evolution, ed R. L. M. Corradi, J. Mikołajewska \& T. J. Mahoney (San Francisco, CA: ASP), 9

Mikołajewska, J. 2011, in IAU Symp. 281, Symbiotic Stars as Possible Progenitors of SNe Ia: Binary Parameters and Overall Outlook, ed. R. di Stefano, M. Orio \& M. Moe (Cambridge: Cambridge Univ. Press), 162

Mikołajewska, J. 2012, BaltA, 21, 5

Mikołajewska, J., Kenyon, S. J., \& Mikołajewska, M. 1989, AJ, 98, 1427

Miszalski, B., \& Mikołajewska, J. 2014, MNRAS, 440, 1410

Miszalski, B., Mikołajewska, J., \& Udalski, A. 2013, MNRAS, 432, 3186

Munari, U., \& Patat, F. 1993, A\&A, 277, 195

Munari, U., Yudin, B. F., Taranova, O. G., et al. 1992, A\&AS, 93, 383
Mürset, U., Dumm, T., Isenegger, S., et al. 2000, A\&A, 353, 952

Mürset, U., \& Schmid, H. M. 1999, A\&AS, 137, 473

Percy, J. R., Wilson, J. B., \& Henry, G. W. 2001, PASP, 113, 983

Pereira, C. B., \& Roig, F. 2009, AJ, 137, 118

Phillips, J. P. 2007, MNRAS, 376, 1120

Pojmanski, G. 2002, AcA, 52, 397

Quiroga, C., Mikołajewska, J., Brandi, E., Ferrer, O., \& Garcia, L. 2002, A\&A, 387,139

Robinson, K., Bode, M. F., Skopal, A., Ivison, R. J., \& Meaburn, J. 1994, MNRAS, 269, 1

Sanduleak, N., \& Stephenson, C. B. 1973, ApJ, 185, 899

Scarfe, C. D., Batten, A. H., \& Fletcher, J. M. 1990, PDAO, 18, 21

Schmid, H. M., \& Nussbaumer, H. 1993, A\&A, 268, 159

Schmid, H. M., \& Schild, H. 2002, A\&A, 395, 117

Schmutz, W., Schild, H., Mürset, U., \& Schmid, H. M. 1994, A\&A, 288, 819

Seaquist, E. R., Krogulec, M., \& Taylor, A. R. 1993, ApJ, 410, 260

Smith, V. V., Cunha, K., Jorissen, A., \& Boffin, H. M. J. 1997, A\&A, 324, 97

Soszyński, I., Dziembowski, W. A., Udalski, A., et al. 2007, AcA, 57, 201

Swings, J. P., \& Allen, D. A. 1972, PASP, 84, 523

Wackerling, L. R. 1970, MmRAS, 73, 153

Webster, B. L., \& Allen, D. A. 1975, MNRAS, 171, 171

Whitelock, P. A. 1987, PASP, 99, 573

Wils, P. 2003, IBVS, 5457, 1

Zahn, J.-P. 1977, A\&A, 57, 383

Zamanov, R. K., Bode, M. F., Melo, C. H. F., et al. 2007, MNRAS, 380, 1053 